\title{
Phytate-hydrolyzing rhizobacteria: abiotic stress tolerance and antimicrobial activity
}

\author{
Daria Itkina $^{1}$, and Aliya Suleimanova ${ }^{1, *}$ \\ ${ }^{1}$ Laboratory of Microbial Biotechnology, Institute of Fundamental Medicine and Biology, Kazan \\ (Volga region) Federal University, 420126 Kazan, Russia
}

\begin{abstract}
Phytate-hydrolyzing bacteria Pantoea sp. 3.1, 3.2, 3.5.2, 3.6.1 and Bacillus ginsengihumi M2.11 were previously isolated from the soil samples of the Republic of Tatarstan. The effect of cultivation conditions on the growth dynamics as well as antimicrobial activity was determined. All four Pantoea strains showed optimum growth at $26^{\circ} \mathrm{C}$ and $28{ }^{\circ} \mathrm{C}$ and $\mathrm{pH}$ 6.0-7.0. The optimum conditions for the growth of $B$. ginsengihumi M2.11 strain was determined to be $26^{\circ} \mathrm{C}, 28^{\circ} \mathrm{C}$ and $37^{\circ} \mathrm{C}$ and alkaline $\mathrm{pH}$ 7 and 8. Salt concentration in the range of 0 to $1000 \mathrm{mM}$ did not significantly affect the growth of the strains. Antagonistic activity of Pantoea sp. 3.5.2 was studied against phytopathogenic micromycetes, identified as Alternaria alternata and Bipolaris sorokiniana. In the presence of bacterial isolate growth of A. alternata was inhibited by $57 \%$ and growth of $B$. sorokiniana - by $85 \%$. Minor growth inhibition by Pantoea sp. 3.5.2 of gram-negative bacteria from Enterobacteriaceae family was observed. The presence of fungicidal activity in the Pantoea strain together with its ability to hydrolyze soil phytates and overcome abiotic stress factors in soil can possibly serve as the basis for the new fungicide of microbial origin.
\end{abstract}

\section{Introduction}

One of the main problems of the new millennium is getting more and more agricultural products from fertile arable land, which is annually reducing per capita. The increasing public health concern, growing organic food industry, environmental pollution with pesticides, eutrophication of water reservoirs due to the massive use of mineral fertilizers, as well as significant increase in their cost have played a huge role in the development of the global biofertilizer industry. Thus, biological fertilizers and biopesticides occupy a separate unique place in the market of agricultural products all over the world today.

A significant improvement of crops growth and yield due to beneficial microorganisms is described by many authors [1-3]. However, insufficient knowledge of the basic molecular mechanisms of that interaction hinders widespread commercial use of plantgrowth-promoting rhizobacteria (PGPR). PGPR are characterized by production of plant growth regulators such as indolyl-3-acetic acid (IAA), nitrogen fixation, mobilization of

* Corresponding author: aliya.kzn@gmail.com 
phosphorus and other macro- and microelements of the soil, and antagonism of plant pathogens by secreting siderophores, cellulases, proteases, antibiotics and cyanide, thus beneficially affecting the plant growth [2].

Increasing the availability of macronutrients for plants' nutrition is an important step in the improvement of agricultural production. In soil, the unavailable form of organic phosphorus is mainly represented by phytic acid salts - phytates. Enzymes that break down phytate phytases - are actively secreted by some soil microorganisms and release inorganic phosphorus from insoluble phytates [4]. There is no extracellular phytase activity of plant origin in the rhizosphere, therefore, microbial phytases play crucial role in the soil organic phosphorus cycle [5].

At the same time, the acute problem in agriculture is the fight against plant diseases caused by phytopathogenic microorganisms. Phytopathogenic micromycetes are capable of releasing biologically active substances that damage plant tissues by slowing down the respiration, reducing photosynthesis, disrupting nitrogen and carbohydrate metabolism, damaging cell membranes, inhibiting mitochondrial DNA replication, and causing disruption of the mitotic cycle in meristems [6]. Moreover, bacteria are also able to cause diseases in a wide range of plants worldwide. Phytopathogenic bacteria affect foodproducing plants, colonizing either their surface or tissues and causing symptoms such as blights, spots, tissue rots, hormone misbalances that globally impact plants growth and development [7].

Therefore, usage of biological preparations based on the bacteria, capable of utilizing the natural bio-resources of the soil, satisfying the nutritional needs of plants together with the protecting them from phytopathogenic microorganism is a promising approach to sustainable agriculture nowadays. Despite numerous positive effects, often the commercialization of bacterial bio-fertilizers on a large scale is limited due to the incompatibility between the results of laboratory studies and field application of the biopreparation [8]. This may be due to a number of factors, such as physicochemical properties of the soil (for example, low or high $\mathrm{pH}$ ), interaction with other rhizospheric organisms, poor ability of the strain to colonize plant roots, environmental factors (high or low temperature, low rainfall during vegetation, high salinity of the soil and etc). The survival of bacteria in the soil, in addition to the presence of empty econiche, depends on the ability of these bacteria to compete with native microorganisms well adapted to given conditions [9].

Earlier, we have isolated phytate-hydrolyzing bacteria from the soil samples of the Republic of Tatarstan, Russia and identified them as Pantoea sp. 3.1, 3.2, 3.5.2 and 3.6.1, and Bacillus ginsengihumi M2.11. The autochthony of rhizosphere microorganisms isolated from the soil of the Republic of Tatarstan prevents the disturbance of the soil agrobiocenosis. Combined with their high efficiency, that makes them a great candidate for the use as biofertilizer in agriculture in this region. In the present work, the effect of cultivation conditions at different temperatures, $\mathrm{pH}$ values and salinity of the medium on the growth dynamics of the isolated rhizosphere strains as well as their antimicrobial activity is determined.

\section{Materials and methods}

\subsection{Bacterial strains and culture media}

Bacterial strains used in this study were previously isolated from the soil samples of the Republic of Tatarstan according to their ability to hydrolyze phytate. Pantoea strains sp. 
3.1, 3.2, 3.5.2 and 3.6.1 were isolated from the forest soil sample and Bacillus ginsengihumi M2.11- from the soil sample of the large farm complex GUP "Mayskiy". These microorganisms were maintained at $4^{\circ} \mathrm{C}$ on Luria-Bertani Agar (LB).

The examined fungi were cultivated in the Czapek medium [10]: Saccharose, $30.0 \mathrm{~g}$; NaNO3, 3.0 g; K2HPO4, 1.0 g; MgSO4.7H2O, 0.5 g; KCl, 0.5 g; FeSO4.7H2O, 0.01 g; agar, $15 \mathrm{~g}$; distilled water, $900 \mathrm{ml} ; \mathrm{pH} 4.5$. The plates were incubated at $28^{\circ} \mathrm{C}$ for $5-14$ days.

\subsection{Effect of salt, $\mathrm{pH}$ and temperature on the bacterial growth}

The effect of different temperature on the growth of Pantoea and B.ginsengihumi M2.11 strains was evaluated in the temperature range from 4 to $40{ }^{\circ} \mathrm{C}$. To determine the effect of $\mathrm{pH}$ on the bacterial growth, $\mathrm{pH}$ range from 2.0 to 9.0 was used. The influence of salt on bacterial growth was determined on $\mathrm{LB}$ medium in the range of $\mathrm{NaCl}$ concentrations from 0 $\mathrm{mM}$ to $1000 \mathrm{mM}$.

The LB-broth was inoculated with $1 \%$ of the culture. Bacteria were grown for $48 \mathrm{~h}$ at $200 \mathrm{rpm}$ shaking. The study was performed in three biological replicates. Growth of the cultures was evaluated by the change in optical density (OD) on a spectrophotometer (BioRad, USA) at a wavelength of $600 \mathrm{~nm}$ every 4 hours.

\subsection{Identification of micromycetes}

DNA was isolated from the mycelium using the Don Liu method [11] [Liu et al., 2000]. The mycelium was thoroughly rubbed in $500 \mathrm{ml}$ of TE buffer $(400 \mathrm{~mm}$ Tris- $\mathrm{HCl}, 60 \mathrm{~mm}$ EDTA, 1\% SDS, $\mathrm{pH} 8.0$ ) to a homogeneous suspension and left for 15 minutes at $25^{\circ} \mathrm{C}$. $150 \mathrm{ml}$ of potassium acetate buffer $(\mathrm{pH} 4.8 ; 60 \mathrm{ml}$ of $5 \mathrm{M}$ of potassium acetate, $11.5 \mathrm{ml}$ of glacial acetic acid, $28.5 \mathrm{ml}$ of distilled water) was added, suspension was shaken and then centrifuged at 11,000 rpm for $1 \mathrm{~min}$. Equal volume of isopropyl alcohol was added and carefully mixed. The mixture was then centrifuged at 11,000 rpm for 2 minutes and supernatant was discarded. The resulting DNA precipitate was washed with $300 \mu 1$ of $70 \%$ ethanol and centrifuged at $11,000 \mathrm{rpm}$ for $1 \mathrm{~min}$, the supernatant was discarded. The precipitate was dried at room temperature and dissolved in $50 \mu 1$ of $1 \times$ Tris-EDTA. Purified DNA was used for PCR.

Amplification and sequencing of ITS (internal transcribed spacer) region was performed to identify intraspecific differences in the sequences of these genes. PCR was carried out using the Taq-polymerase (Thermo Fisher Scientific, USA) with combinations of ITS1ITS4 PCR primer pair (ITS1 dir -tccgtaggtgaacctgcgg; ITS4 rev - tcctcegcttattgatatgc) for amplification of the coding part of 5.8S rRNA ribosomal genes [12]. Samples were incubated for $4 \mathrm{~min}$ at $90^{\circ} \mathrm{C}$, subjected to 35 cycles of denaturation $\left(30 \mathrm{~s}\right.$ at $\left.94^{\circ} \mathrm{C}\right)$, annealing $\left(20 \mathrm{~s}\right.$ at $\left.55^{\circ} \mathrm{C}\right)$, and extension $\left(40 \mathrm{~s}\right.$ at $\left.72^{\circ} \mathrm{C}\right)$, followed by a final extension step ( 7 min at $72^{\circ} \mathrm{C}$ ). Presence of PCR products was confirmed by DNA electrophoresis in $1 \%$ agarose gel. PCR products were purified using a PCR purification kit (Thermo Scientific, Lithuania) and sequenced at Syntol (Moscow, Russia). The analysis of 5.8S rRNA gene sequences was performed using the BLAST algorithm, a software package presented on the NCBI server (http://www.ncbi.nlm.nih.gov/blast). 


\subsection{In vitro antifungal activity assay}

To determine the antagonistic activity of Pantoea strain against the micromycetes the agar well method was used [13]. Micromycetes were cultured on Czapek medium for 7 days. Then the suspension of mycelial and spore mass $\left(10^{5} \mathrm{CFU} / \mathrm{ml}\right)$ in LB broth was prepared from the micromycete colonies. Pantoea strain was cultured on LB-agar plates for $24 \mathrm{~h}$, then $8 \mathrm{~mm}$ diameter well was excised from the centre of the agar plate and $200 \mu \mathrm{l}$ of micromycete suspension $\left(10^{5} \mathrm{CFU} / \mathrm{ml}\right)$ was added. Control plates without bacterial growth were prepared simultaneously. Plates were incubated at $28^{\circ} \mathrm{C}$ for $7-14$ days and examined for the growth inhibition. Experiments were repeated three times. The growth inhibition of the test fungi was calculated using the following formula:

Inhibition $(\%)=(\mathrm{R}-\mathrm{r}) / \mathrm{R} * 100$,

where $\mathrm{R}$ - (a control value) represents the radial growth of fungus in control sets. $\mathrm{r}$ - the radial growth of the fungus in sets with bacteria.

\subsection{Screening of antibacterial activities of isolates}

\subsubsection{Perpendicular streak method}

Screening for the antibacterial effect of Pantoea sp. 3.5.2 was carried out by perpendicular streak method of Madigan et al. [14] against the following strains: Serratia marcescens, $S$. grimesii, Morganella morganii, Erwinia sp., Pantoea agglomerans, E. coli dH5a, Salmonella enterica and Bacillus pumilus. A single streak of the pure producer strain was inoculated in the middle of the assay LB-plate. The plates were incubated for 4 days at $37^{\circ} \mathrm{C}$. After the incubation period, test organisms were cross-streaked along the line of grown isolate and finally the plates were incubated for $1-2$ days at $37^{\circ} \mathrm{C}$. Each streaking was started near the edge of the plates and streaked toward the Pantoea growth line. The microbial interactions were analysed by evaluating the zones of inhibition measured in $\mathrm{mm}$.

\subsection{Preparation of cellular extract}

Cells were grown in LB medium at $37^{\circ} \mathrm{C}$ for $20 \mathrm{~h}$ and harvested at $4{ }^{\circ} \mathrm{C}$ by centrifugation for $10 \mathrm{~min}$ at $8000 \mathrm{~g}$. Cells were then washed 3 times with $20 \mathrm{mM}$ sodium acetate buffer, $\mathrm{pH} 4.5$, and lysed 3 times by repeated freezing at $80^{\circ} \mathrm{C}$ for $15 \mathrm{~min}$ and thawing at room

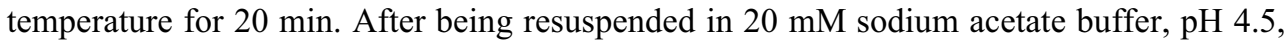
cells were sonicated on ice 10 times for $10 \mathrm{~s}$ with 30 -s intervals. Cellular debris was removed by centrifugation at $4^{\circ} \mathrm{C}$ for $30 \mathrm{~min}$ at $18,000 \mathrm{rpm}$.

\subsubsection{Determination of Minimum Inhibitory Concentrations (MIC)}

The inhibitory potential of bacteria growth by culture liquid and cell extract of Pantoea sp. was determined by broth microdilution method in 96-well microtiter plates [15]. Escherichia coli Dh5 $\alpha$ and Serratia marcescens Sm6 strains were used as test cultures. The inoculum of the strains was diluted 1000 times in LB and cultured at $37^{\circ} \mathrm{C}$ until the OD600 $0.1\left(10^{8} \mathrm{CFU} / \mathrm{ml}\right)$ was reached. For the experiment, microbial cultures were diluted to a concentration of $1000 \mathrm{CFU} / 10 \mu \mathrm{l}$. Wells of a 96-well plate were treated with sterile $0.1 \%$ BSA for $10 \mathrm{~min}$ at room temperature. $90 \mu$ of PBS buffer was then added to each well. As the active substance, $90 \mu \mathrm{l}$ of cell extract (CE) or culture liquid (CL) of Pantoea were added to the first wells of a row of a 96-well plate and a serial 10-fold 
dilution was performed. Ampicillin $(100 \mu \mathrm{g} / \mathrm{ml})$ was used as a control. The plate was incubated at $37^{\circ} \mathrm{C}$ for 12 hours and the results were evaluated. MIC values were defined as the sample concentration that prevented the bacterial growth [16].

\section{Results and discussion}

\subsection{Responses of the strains to Abiotic Stress factors}

One of the strategies to overcome the limitations of biofertilizers application is the use of native microorganisms adapted to the ecological conditions of each region [17]. We have isolated phytate-hydrolyzing bacteria from various agro-ecological niches and natural habitats of the Republic of Tatarstan, Russia $[18,19]$. Strains were isolated on PSM solid medium, which contains calcium phytate as a sole source of phosphorus. The maximum number of phytate-hydrolyzing colonies was obtained from forest soils, which had more than $100 \times 10^{3} \mathrm{CFU}$ per gram of soil. Soil of large farm "Mayskiy" contained $29 \times 10^{3}$ colony forming units (CFU) per gram of soil. Isolates were identified as Pantoea sp. 3.1, 3.2, 3.5.2, 3.6.1 and B.ginsengihumi M2.11.

Plant growth is strongly influenced by many biotic and abiotic factors, which limit the use of biofertilizers [7]. Investigation of the role of temperature, $\mathrm{pH}$ and salinity on the growth of isolated soil bacteria was performed. All four Pantoea strains showed similar response to tested factors, so the average data is present on the figures.

We examined the effect of temperature in the range of $4-40^{\circ} \mathrm{C}$ on the growth dynamics of Pantoea strains (Fig. 1A) and B. ginsengihumi M2.11 strain (Fig. 1B). The optimum temperatures for the growth of Pantoea strains were $26{ }^{\circ} \mathrm{C}$ and $28{ }^{\circ} \mathrm{C}$ (Fig. 1A) - cultures reached the maximum level of biomass compared to growth at other temperatures. Bacteria entered the prolonged stationary phase at 12 hour of growth. Growth curve of Pantoea strains at $37^{\circ} \mathrm{C}$ was comparable or even better at the initial stage of growth, but after 16 hours a sharp decrease in the level of biomass accumulation occurred. This is explained by the fact that the temperature typical for the body of mammals (around $37^{\circ} \mathrm{C}$ ) is not optimal for the growth of strains isolated from soil ecosystem. At $4{ }^{\circ} \mathrm{C}$ the ability to grow was practically inhibited. B. ginsengihumi M2.11 strain showed same high level of biomass accumulation at $26^{\circ} \mathrm{C}, 28^{\circ} \mathrm{C}$ and $37^{\circ} \mathrm{C}$ (Fig.1B). Bacteria entered the stationary phase only at 20 hour of growth. Growth at $4{ }^{\circ} \mathrm{C}$ was almost inhibited and significantly reduced at $40^{\circ} \mathrm{C}$. The obtained data indicate that all tested Pantoea strains and B. ginsengihumi M2.11 strain are mesophilic.
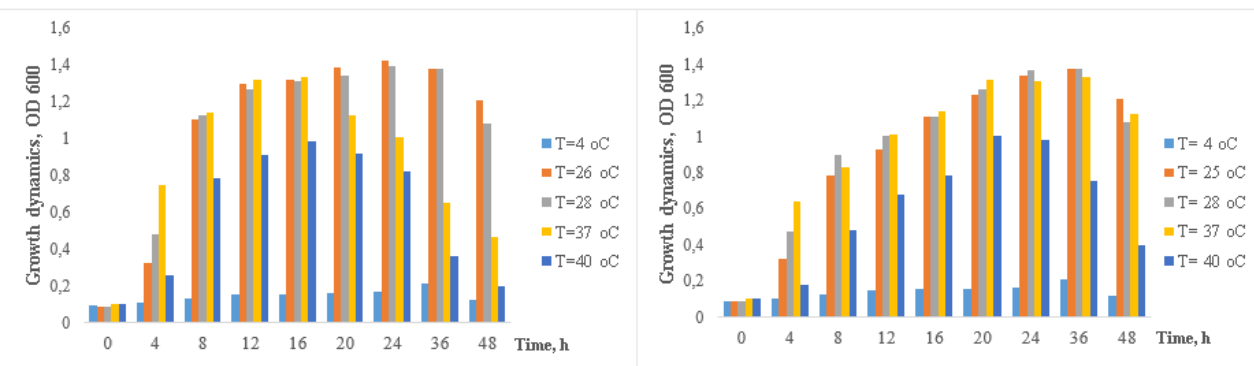
A B

Fig.1. Growth dynamics of Pantoea sp. (A) and B. ginsengihumi M2.11 (B) strains at different temperatures. 
The effect of medium acidity on the growth of isolated bacterial strains was studied (Fig. 2). It was shown that all Pantoea strains were able to grow in the range of $\mathrm{pH}$ values from 6 to 9 (Fig. 2A). The response to cultivation under acidic $\mathrm{pH}$ conditions $(2.0-5.0)$ was expressed by almost complete growth inhibition. The optimal $\mathrm{pH}$ for the cultivation of Pantoea strains was $\mathrm{pH}$ 6.0-7.0. The biomass accumulation of the Pantoea strains at $\mathrm{pH} 9.0$ was lower than at optimal $\mathrm{pH}$ values. B. ginsengihumi M2.11 demonstrated great growth ability over a broad $\mathrm{pH}$ range, ranging from $\mathrm{pH} 6.0$ to $\mathrm{pH} 9.0$. There was inhibition of growth at acidic $\mathrm{pH}$ values $(\mathrm{pH} 2.0-5.0)$. The bacteria showed optimum growth at $\mathrm{pH} 7.0$ 8.0 (Fig. 2B).

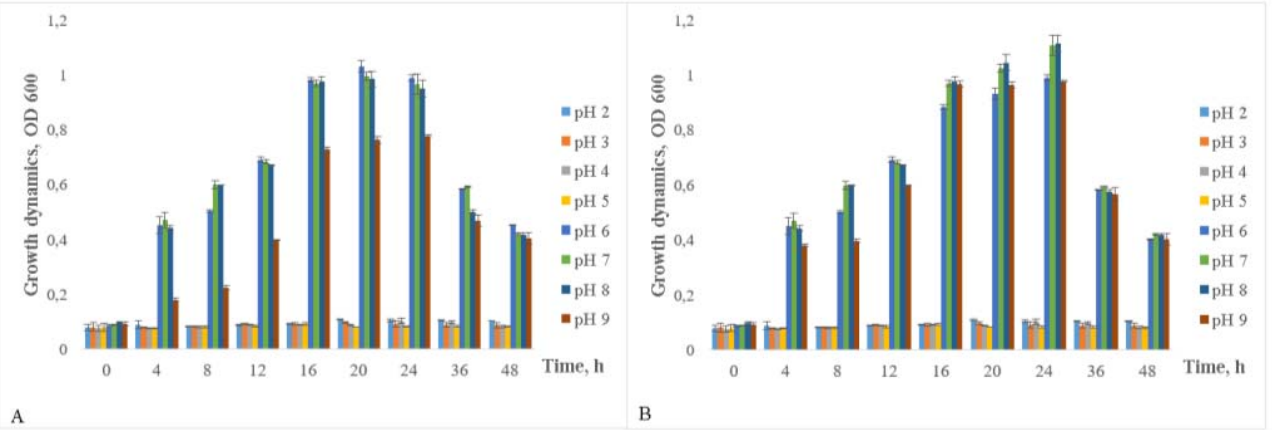

Fig.2. Growth dynamics of Pantoea sp. (A) and B. ginsengihumi M2.11 (B) strains at different pH values of the medium.

Soil salinity plays an important role in the microbial selection process [20]. Growth of all Pantoea sp. and B. ginsengihumi M2.11 strains in the nutrient broth with variations in salt concentration from 0 to $1000 \mathrm{mM}$ showed a high tolerance capacity of the strains to high salt levels in the medium (Fig. 3). At the early stage of growth (4h) $\mathrm{NaCl}$ concentrations higher than $500 \mathrm{mM}$ held back the growth of bacteria. Nevertheless, by the $24^{\text {th }}$ hour of cultivation the level of biomass accumulation by all tested isolates remained high at all salt concentrations (Fig. 3). Thus, salt concentration of the medium did not significantly affect the growth of neither Pantoea strains nor B. ginsengihumi M2.11. Still, Pantoea strains showed slightly higher salt tolerance than B. ginsengihumi M2.11.
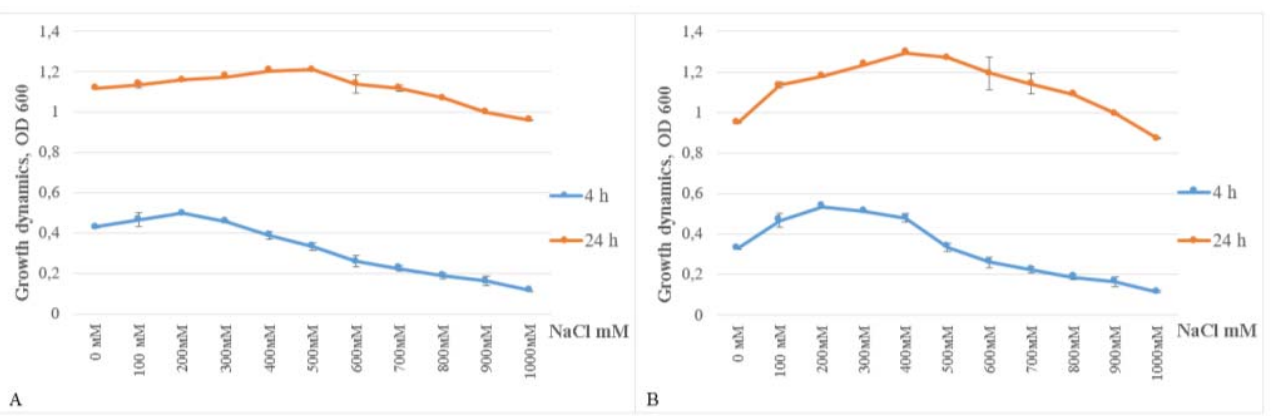

Fig.3. Effect of different concentrations of $\mathrm{NaCl}(\mathrm{mM})$ on the growth of Pantoea sp. (A) and $B$. ginsengihumi M2.11 (B) strains at $4 \mathrm{~h}$ and $24 \mathrm{~h}$ of incubation. 


\subsection{Identification of micromycetes and antagonistic activity of Pantoea sp. 3.5.2 in dual culture}

Antagonistic activity of Pantoea sp. 3.5.2 was studied against phytopathogenic micromycetes provided by the Tatar Research Institute of Agriculture of the Agricultural Academy. Two fungal isolates were isolated from the infected plants. For precise genetic identification of fungal pathogens we used molecular genetic markers representing nucleotide sequences of the ITS regions [12]. A significant part of the ITS regions is represented by conserved sequences with identical nucleotide composition in various microorganisms, and species-specific variable regions, which allow to identify the species by comparing these sequences with those annotated in the databases [21]. Amplification of the 5.8S rRNA gene of isolated micromycetes was performed with standard primers ITS1 and ITS4 (Fig. 4).

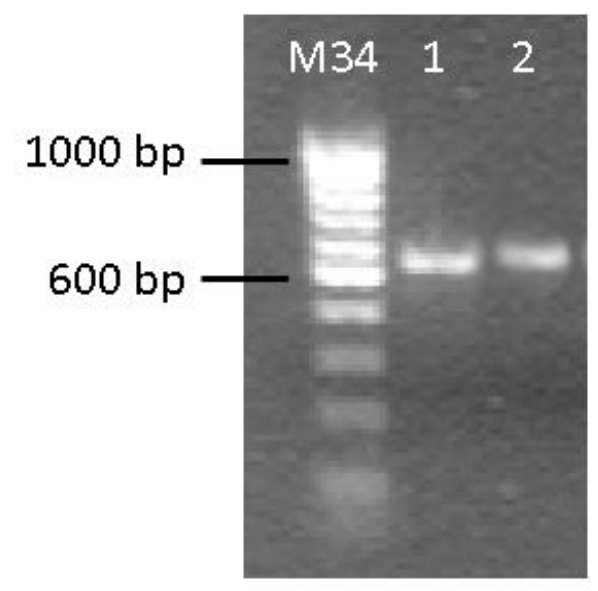

Fig. 4. PCR amplification of ITS (internal transcribed spacer) region of the coding part of 5.8S rRNA ribosomal genes. Lane M 34: molecular weight markers, 1000 bp, Lane1 - Isolate 1, Lane2 - Isolate 2.

Sequencing of amplified ITS regions allowed to identify the high homology of isolates to the Alternaria alternata KS44T14 and Bipolaris sorokiniana HP-1744 - the identity of the sequences was $99 \%$. Due to their wide host range and worldwide distribution, Alternaria species cause severe economic problems. A. alternata is able to produce diverse phytotoxins. A. alternata infection in leaves induces rapid lipid peroxidation, accumulation of hydrogen peroxide $\left(\mathrm{H}_{2} \mathrm{O}_{2}\right)$, and cell death [22]. B. sorokiniana is the causal agent of common root rot, leaf spot and seedling blight, head blight of wheat and barley and black point of grains [23]. Both fungal isolates posses high risk for the quality and yield of economically valuable cultures. Therefore, constant search of new antagonistic bacteria against these fungi is performed.

Pantoea strain 3.5.2 was screened for antagonistic activity by measuring the inhibition zones present after 7 days of dual culture with phytopathogenic fungi. Using the PetatanSagahon well method on LB agar medium we showed that Pantoea sp. 3.5.2 had the ability to inhibit the growth of both A. alternata and B. sorokiniana (Fig. 5). Growth of $A$. alternata was inhibited by $57 \%$ in the presence of bacterial isolate and growth of $B$. sorokiniana - by $85 \%$. In contrast, no inhibition zone was observed in the control plate, where fungal mycelia covered almost the entire plate surface (Fig. 5). 

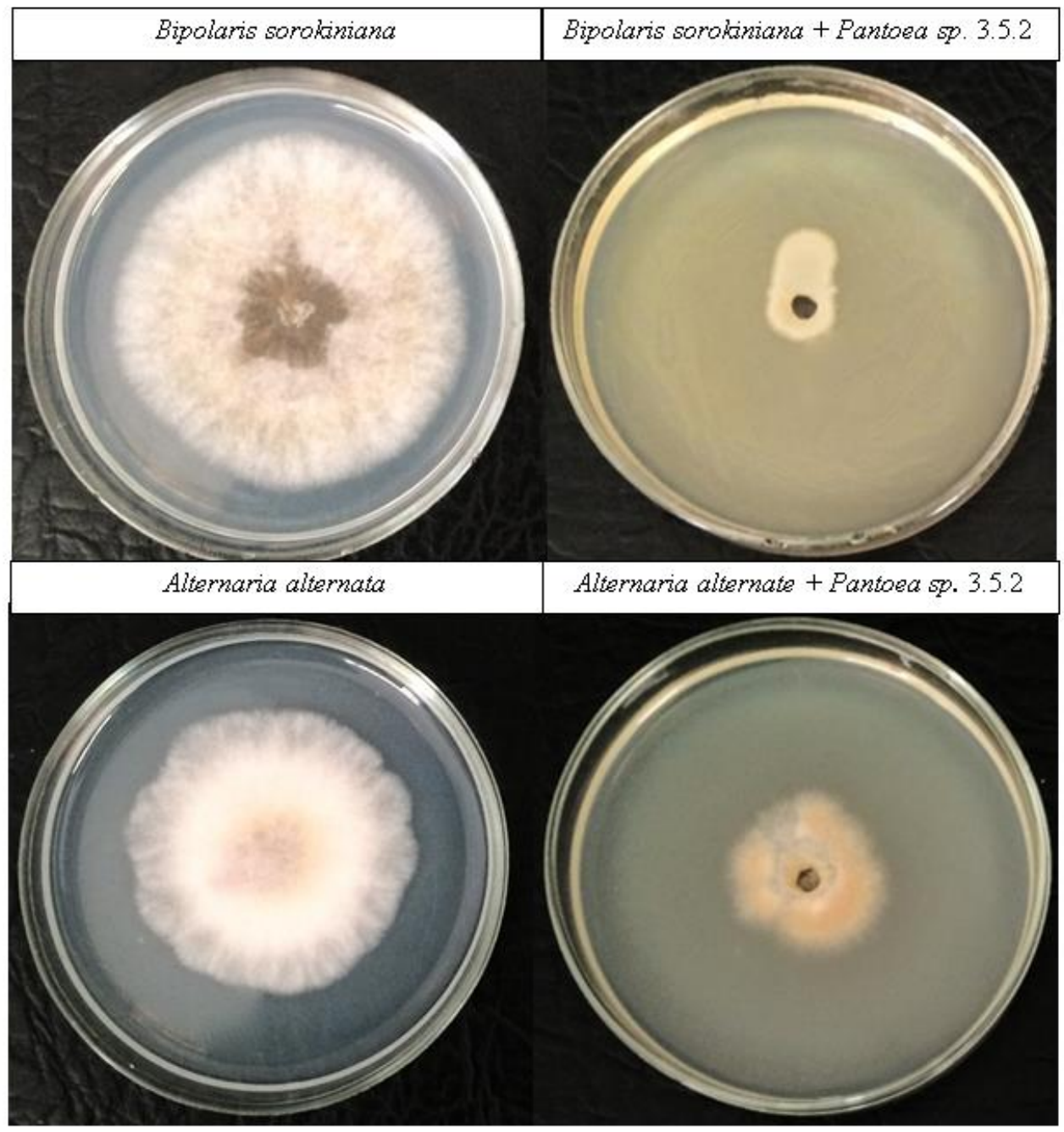

Fig.5. Antagonism of Pantoea sp. 3.5.2 against fungal phytopathogens - A. alternata and $B$. sorokiniana. Growth inhibition of fungal mycelia was examined in dual culture assay on agar plates.

Thus, we observed changes in the mycelium morphology caused by bacteria in comparison to the control - irregular and distorted shape appeared. Such alteration of mycelium morphology in the presence of bacterial metabolites was reported by many authors [24], [25]. Antifungal action of Pantoea may be a result of disruption of fungal cell wall or inhibition of normal conidia development [26]. It was shown, that Pantoea strain produced compounds with fungicidal activity against Alternaria and Bipolaris genera during its growth. The presence of fungicidal activity in the Pantoea strain together with its ability to hydrolyze soil phytates and overcome abiotic stress factors in soil can possibly serve as the basis for the new fungicide of microbial origin. 


\subsection{Antibacterial Activities of Pantoea sp. 3.5.2}

Isolated Pantoea sp. 3.5.2 strain was screened for its antibacterial activity on LB-agar medium using streak-plating technique (Fig. 6). No inhibition zones were detected between Pantoea sp. 3.5.2 and three test-strains: gram-positive Bacillus pumilus 3-19 strain and the strains of the same genus as the producer-strain $-P$. ananatis $\mathrm{Cl}-18$ and P. agglomerans U2-22. Minor growth inhibition of gram-negative bacteria from Enterobacteriaceae family - S. enterica, S. marcescens Sm6, S. grimesia, M. morganii, E. coli Dh5 $\alpha$ - was observed (Fig. 6).
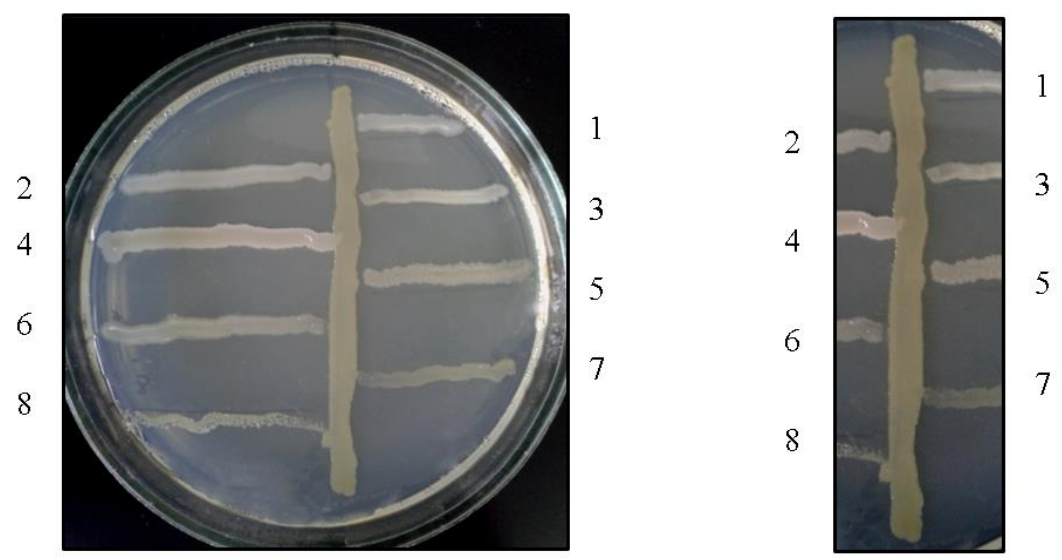

Fig. 6. Streak-plating technique to screen the antibacterial activity of isolated Pantoea sp. 3.5.2.: 1 $S$. enterica, 2 - S. marcescens Sm6, 3 - S. grimesia, 4 - Bacillus pumilus 3-19, 5 - M. Morganii, 6 E. coli $\mathrm{Dh} 5 \alpha, 7$ - P. ananatis $\mathrm{Cl}-18,8$ - P. agglomerans $\mathrm{U} 2-22$.

To determine the minimum inhibitory concentration of the antimicrobial compound synthesized by Pantoea sp. 3.5.2, two bacterial cultures were used: E. coli Dh5 $\alpha$ and $S$. marcescens Sm6. Since the localization and nature of the antimicrobial compound is unknown, we studied both culture liquid (CL) and cell extract (CE) of the strain for antibacterial activity. The growth of $E$. coli was suppressed by CL at the concentration of $10^{-5}$, and the growth of $S$. marcescens - in the concentration of $10^{-7}$. While studying the effect of the CE of Pantoea sp. 3.5.2 on bacterial growth, it was found that MIC to inhibit the growth of $E$. coli and $S$. marcescens is $10^{-9}$ and $10^{-8}$, respectively. The inhibitory concentration of the control antibiotic (ampicillin), causing complete inhibition of bacterial growth, was $10^{-5}(16 \mu \mathrm{g} / \mathrm{ml})$ for $E$. coli and $10^{-7}(12 \mu \mathrm{g} / \mathrm{ml})$ for $S$. marcescens. In the control rows, microorganism growth was observed in all wells (Fig. 7). 


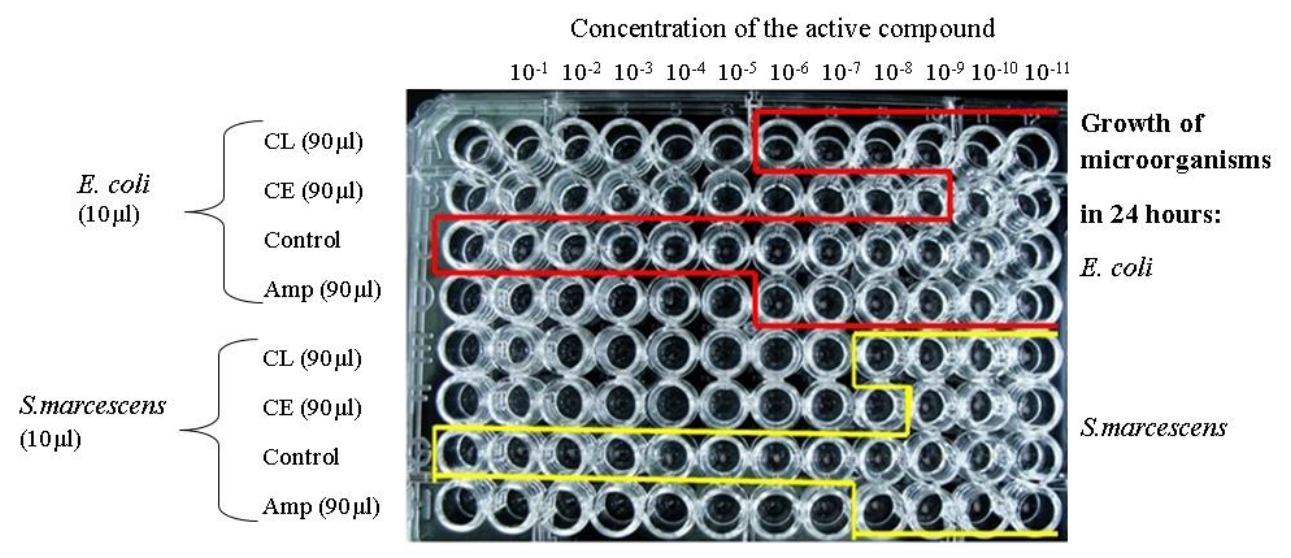

Fig.7. Checkerboard microtiter plate assay testing the antibacterial activity of culture liquid (CL) and cell extract (CE) of Pantoea sp. 3.5.2 against E. coli and S. marcescens.

Thus, it was found that the antibacterial compound of Pantoea sp. 3.5.2 is present both in the culture fluid and in the cell extract of the strain.

\section{Conclusion}

Phytate-hydrolyzing bacteria Pantoea sp. 3.1, 3.2, 3.5.2, 3.6.1 and Bacillus ginsengihumi M2.11 were previously isolated from the soil samples of the Republic of Tatarstan. In order to understand their competitive ability in soil and survival under different environmental conditions we studied the effect of abiotic factors on their growth. As a result of experiments, we were able to demonstrate that all four Pantoea strains showed optimum growth at $26^{\circ} \mathrm{C}$ and $28{ }^{\circ} \mathrm{C}$ and $\mathrm{pH} 6.0-7.0$. The optimum conditions for the growth of $B$. ginsengihumi M2.11 strain was determined to be $26^{\circ} \mathrm{C}, 28^{\circ} \mathrm{C}$ and $37^{\circ} \mathrm{C}$ and alkaline pH 7 and 8 . Salt concentration in the cultivation medium in the range of 0 to $1000 \mathrm{mM}$ did not significantly affect the growth of neither Pantoea strains, nor B. ginsengihumi. Antagonistic activity of Pantoea sp. 3.5.2 was studied against phytopathogenic micromycetes, identified as Alternaria alternata and Bipolaris sorokiniana. Growth of $A$. alternata was inhibited by $57 \%$ in the presence of bacterial isolate and growth of B. sorokiniana - by $85 \%$. Minor growth inhibition by Pantoea sp. 3.5.2 of gram-negative bacteria from Enterobacteriaceae family - S. enterica, S. marcescens Sm6, S. grimesia, M. мorganii, E. coli Dh5a - was observed.

Thus, biological preparation based on phytate-hydrolyzing bacteria may be used as a new environmentally friendly biofertilizer, capable of utilizing the natural bio-resources of the soil, satisfying the nutritional needs of plants and protecting them from phytopathogenic micromycetes.

\section{Acknowledgments}

This work was supported by the Russian Science Foundation (Project No. 19-76-00020) and was performed in accordance with the Russian Government Program of Competitive Growth of Kazan Federal University. 


\section{References}

1. L. Arruda, A. Beneduzi, A. Martins, B. Lisboa, C. Lopes, F. Bertolo, Agric. Ecosyst. Environ. Appl. Soil Ecol., 63, 15-22 (2013)

2. R. Singh, P. Singh, R. Sharma, Article Proceedings of the International Academy of Ecology and Environmental Sciences, 4(1), 1-6 (2014)

3. L. Liu, S. Oza, D. Hogan, Y. Chu, J. Perin, J. Zhu, The Lancet ARTICLES, 388, 3027-3035 (2016)

4. M. D. Mullen, Encyclopedia of Soils in the Environment. Elsevier Ltd., Oxford, 210215 (2016)

5. B. Singh, T. Satyanarayana, Physiol Mol Biol Plants, 17(2), 93-103 (2011)

6. S. Timmusk, L. Behers, J. Muthoni, A. Muraya, A. Aronsson, Front Plant Sci., 8, 49 (2017)

7. V.M. Martins, M.M. Paula, M.A. Takita, A.A. De Souza, Front Microbiol., 9, 1099 (2018)

8. M.E. Lucy, E. Reed, B.R. Glick, SourcePubMed Antonie van Leeuwenhoek, 86, 1-25 (2004)

9. H. Etesami, G.A. Beattie, Front Microbiol, 89, 148 (2018)

10. K.B. Raper, D.I. Fennell, The Genus Aspergillus (Williams and Wilkins, Philadelphia, 1965)

11. D.Liu, J. Clin. Microbiol., 38, (2000)

12. T.J. White, T.D. Bruns, S.B. Lee, J.W. Taylor M.A. Innis, A Guide to Methods and Applications (Academic Press: San Diego, CA, USA, 1990)

13. M. Balouiri, M. Sadiki, S.K. Ibnsouda, Pharm Anal., 6(2), 71-79 (2016)

14. M.T. Madigan, J.M. Martiko, J. Parker Brock Biology of Microorganisms, 440-442 (1997)

15. M. Elshikh, S. Ahmed, S. Funston, P. Dunlop, M. McGaw, R. Marchant, I.M. Banatcorresponding, Biotechnol Lett, 38, 1015-1019 (2016)

16. J.M. Andrews, J Antimicrob Chemother, 1, 48, (2001)

17. E. Morales, M. Bustamante, J.R. Gonzalez, M. Guxens, M. Torrent, M. Mendez, R. Garcia-Esteban, J. Julvez, J. Forns, M. Vrijheid, C. Molto-Puigmarti, C. LopezSabater, X. Estivill, J. Sunyer, PLoS One., 6(2) (2011)

18. A.D. Suleimanova, A. Beinhauer, L.R. Valeeva, I.B. Chastukhina, N.P. Balaban, E.V. Shakirov, R. Greiner, M.R. Sharipova, Applied and Environmental Microbiology, 81(19), 6790-6799 (2015)

19. A.A. Akhmetova, A.D. Suleimanova, A.A. Toymentseva, N.P. Balaban, D.L. Iljukhina, M.R. Sharipova, Research Journal of Pharmaceutical, Biological and Chemical Sciences, 6(4), 117-122 (2015)

20. K. Sudhir, S.K. Maurya, D.P. Singh, Indian Journal of Scientific Research, 03(2), 7378 (2012)

21. F. Pereira, J. Carneiro, R. Matthiesen, B. Asch, N. Pinto, L. Gusmao, A. Amorim, Nucleic Acids Res., 38(22) (2010)

22. K.R. Chung, Scientifica (Cairo). 635431 (2012) 
23. J. Kumar, P. Schafer, R. Huckelhoven, G. Langen, H. Baltruschat, E. Stein, S. Nagarajan, K.H. Kogel, Mol Plant Pathol., 1, 3(4), 185-95 (2002)

24. I. Petatan-Sagahon, M.A. Anducho-Reyes, H.V. Silva-Rojas, A. Arana-Cuenca, A. Tellez-Jurado, I.O. Cardenas-Alvarez, Y. Mercado-Flores, International Journal of Molecular Sciences, 12, 5522-5537 (2011)

25. S.Y. Oh, M.S. Park, Y.W. Lim, Microorganisms, 7(6), 169 (2019)

26. T.K. Mazu, B.A. Bricker, H. Flores-Rozas, S.Y. Ablordeppey, Mini Rev Med Chem, 16(7), 555-578 (2016) 